\title{
Simple Graphs as Simplicial Complexes: the Mycielskian of a Graph ${ }^{1}$
}

\author{
Piotr Rudnicki \\ University of Alberta \\ Edmonton, Canada
}

\author{
Lorna Stewart \\ University of Alberta \\ Edmonton, Canada
}

\begin{abstract}
Summary. Harary [10, p. 7] claims that Veblen [20, p. 2] first suggested to formalize simple graphs using simplicial complexes. We have developed basic terminology for simple graphs as at most 1-dimensional complexes.

We formalize this new setting and then reprove Mycielski's [12] construction resulting in a triangle-free graph with arbitrarily large chromatic number. A different formalization of similar material is in [15].
\end{abstract}

MML identifier: SCMYCIEL, version: $\underline{7.12 .02 \quad 4.181 .1147}$

The papers [5], [1], [4], [16], [14], [6], [9], [18], [7], [15], [2], [11], [3], [17], [13], [19], and [8] provide the terminology and notation for this paper.

\section{Preliminaries}

One can prove the following propositions:

(1) For all sets $x, X$ holds $\langle x, X\rangle \notin X$.

(2) For all sets $x, X$ holds $\langle x, X\rangle \neq X$.

(3) For all sets $x, X$ holds $\langle x, X\rangle \neq x$.

(4) For all sets $x_{1}, y_{1}, x_{2}, y_{2}, X$ such that $x_{1}, x_{2} \in X$ and $\left\{x_{1},\left\langle y_{1}, X\right\rangle\right\}=$ $\left\{x_{2},\left\langle y_{2}, X\right\rangle\right\}$ holds $x_{1}=x_{2}$ and $y_{1}=y_{2}$.

(5) For all sets $X, v$ such that $3 \subseteq \overline{\bar{X}}$ there exist sets $v_{1}, v_{2}$ such that $v_{1}$, $v_{2} \in X$ and $v_{1} \neq v$ and $v_{2} \neq v$ and $v_{1} \neq v_{2}$.

(6) For every set $x$ holds $S_{\{x\}}=\{\{x\}\}$.

${ }^{1}$ This work has been partially supported by the NSERC grant OGP 9207.

(C) 2012 University of Białystok CC-BY-SA License ver. 3.0 or later ISSN $1426-2630(\mathrm{p}), 1898-9934(\mathrm{e})$ 
Let us observe that there exists a finite sequence which is finite-yielding.

The following proposition is true

(7) Let $X$ be a non empty finite set and $P$ be a partition of $X$. If $\overline{\bar{P}}<\overline{\bar{X}}$, then there exist sets $p, x, y$ such that $p \in P$ and $x, y \in p$ and $x \neq y$.

Let us note that $\bigcup\{\emptyset\}$ is empty.

Next we state three propositions:

(8) For every set $x$ holds $\bigcup\{\emptyset,\{x\}\}=\{x\}$.

(9) For every set $X$ and for every subset $s$ of $X$ such that $s$ is 1-element there exists a set $x$ such that $x \in X$ and $s=\{x\}$.

(10) For every set $X$ holds $\overline{\overline{\{\{X,\langle x, X\rangle\} ; x \text { ranges over elements of } X: x \in X\}}}=\overline{\bar{X}}$.

Let $G$ be a set. The functor PairsOf $G$ yielding a subset of $G$ is defined as follows:

(Def. 1) For every set $e$ holds $e \in$ PairsOf $G$ iff $e \in G$ and $\overline{\bar{e}}=2$.

The following propositions are true:

(11) For every set $X$ and for every set $e$ such that $e \in$ PairsOf $X$ there exist sets $x, y$ such that $x \neq y$ and $x, y \in \bigcup X$ and $e=\{x, y\}$.

(12) For all sets $X, x, y$ such that $x \neq y$ and $\{x, y\} \in X$ holds $\{x, y\} \in$ PairsOf $X$.

(13) For all sets $X, x, y$ such that $\{x, y\} \in$ PairsOf $X$ holds $x \neq y$ and $x$, $y \in \bigcup X$.

(14) For all sets $G, H$ such that $G \subseteq H$ holds PairsOf $G \subseteq$ PairsOf $H$.

(15) For every finite set $X$ holds $\overline{\overline{\{\{x,\langle y, \bigcup X\rangle\} ; x \text { ranges over elements of } \bigcup X, y \text { ranges over elements of }}}$ $\overline{\bar{U} X:\{x, y\} \in \text { PairsOf } X\}}=2 \cdot \overline{\text { PairsOf } X}$.

(16) For every finite set $X$ holds $\overline{\{\langle x, y\rangle ; x \text { ranges over elements of } \bigcup X, y \text { ranges over elements of }}$ $\overline{\overline{U X:\{x, y\} \in \text { PairsOf } X\}}}=2 \cdot \overline{\overline{\text { PairsOf } X}}$.

Let $X$ be a finite set. Note that PairsOf $X$ is finite.

Let $X$ be a set. We say that $X$ is void if and only if:

(Def. 2) $\quad X=\{\emptyset\}$.

One can verify that there exists a set which is void.

Let us observe that every set which is void is also finite.

Let $G$ be a void set. Observe that $\bigcup G$ is empty.

Next we state two propositions:

(17) For every set $X$ such that $X$ is void holds PairsOf $X=\emptyset$.

(18) For every set $X$ such that $\bigcup X=\emptyset$ holds $X=\emptyset$ or $X=\{\emptyset\}$.

Let $X$ be a set. We say that $X$ is pair free if and only if: 
(Def. 3) PairsOf $X$ is empty.

We now state the proposition

(19) For all sets $X, x$ such that $\overline{\overline{U X}}=1$ holds $X$ is pair free.

Let us observe that there exists a set which is finite-membered and non empty.

Let $X$ be a finite-membered set and let $Y$ be a set. Observe that $X \cap Y$ is finite-membered and $X \backslash Y$ is finite-membered.

\section{Simple Graphs as Simplicial Complexes}

Let $n$ be a natural number and let $X$ be a set. We say that $X$ is at most $n$-dimensional if and only if:

(Def. 4) For every set $x$ such that $x \in X$ holds $\overline{\bar{x}} \subseteq n+1$.

Let $n$ be a natural number. Observe that every set which is at most $n$ dimensional is also finite-membered.

Let $n$ be a natural number. Observe that there exists a set which is at most $n$-dimensional, subset-closed, and non empty.

Next we state two propositions:

(20) For every subset-closed non empty set $G$ holds $\emptyset \in G$.

(21) Let $n$ be a natural number, $X$ be an at most $n$-dimensional set, and $x$ be an element of $X$. If $x \in X$, then $\overline{\bar{x}} \leq n+1$.

Let $n$ be a natural number and let $X, Y$ be at most $n$-dimensional sets. Note that $X \cup Y$ is at most $n$-dimensional.

Let $n$ be a natural number, let $X$ be an at most $n$-dimensional set, and let $Y$ be a set. Note that $X \cap Y$ is at most $n$-dimensional and $X \backslash Y$ is at most $n$-dimensional.

Let $n$ be a natural number and let $X$ be an at most $n$-dimensional set. Observe that every at most $n$-dimensional set is at most $n$-dimensional.

Let $s$ be a set. We say that $s$ is simple graph-like if and only if:

(Def. 5) $s$ is at most 1-dimensional, subset-closed, and non empty.

Let us note that every set which is simple graph-like is also at most 1dimensional, subset-closed, and non empty and every set which is at most 1dimensional, subset-closed, and non empty is also simple graph-like.

The following proposition is true

(22) $\{\emptyset\}$ is simple graph-like.

One can verify that $\{\emptyset\}$ is simple graph-like.

One can verify that there exists a set which is simple graph-like.

A simple graph is a simple graph-like set.

One can verify that there exists a simple graph which is void and there exists a simple graph which is finite. 
Let $G$ be a set. We introduce Vertices $G$ as a synonym of $\bigcup G$. We introduce Edges $G$ as a synonym of PairsOf $G$.

Let $X$ be a set. We introduce $X$ is edgesless as a synonym of $X$ is pair free. We now state three propositions:

(23) For every simple graph $G$ such that Vertices $G$ is finite holds $G$ is finite.

(24) For every simple graph $G$ and for every set $x$ holds $x \in \operatorname{Vertices} G$ iff $\{x\} \in G$.

(25) For every set $x$ holds $\{\emptyset,\{x\}\}$ is a simple graph.

Let $X$ be a finite finite-membered set. The functor order $X$ yielding a natural number is defined by:

(Def. 6) $\operatorname{order} X=\overline{\overline{U X}}$.

Let $X$ be a finite set. The functor size $X$ yielding a natural number is defined by:

(Def. 7) size $X=\overline{\overline{\text { PairsOf } X}}$.

Next we state the proposition

(26) For every finite simple graph $G$ holds order $G \leq \overline{\bar{G}}$.

Let $G$ be a simple graph. A vertex of $G$ is an element of Vertices $G$. An edge of $G$ is an element of Edges $G$.

The following propositions are true:

(27) For every simple graph $G$ holds $G=\{\emptyset\} \cup S_{(\text {Vertices } G)} \cup \operatorname{Edges} G$.

(28) For every simple graph $G$ such that Vertices $G=\emptyset$ holds $G$ is void.

(29) Let $G$ be a simple graph and $x$ be a set. If $x \in G$ and $x \neq \emptyset$, then there exists a set $y$ such that $x=\{y\}$ and $y \in \operatorname{Vertices} G$ or $x \in \operatorname{Edges} G$.

(30) For every simple graph $G$ and for every set $x$ such that Vertices $G=\{x\}$ holds $G=\{\emptyset,\{x\}\}$.

(31) For every set $X$ there exists a simple graph $G$ such that $G$ is edgesless and Vertices $G=X$.

Let $G$ be a simple graph and let $v$ be an element of Vertices $G$. The functor Adjacent $(v)$ yielding a subset of Vertices $G$ is defined by:

(Def. 8) For every element $x$ of Vertices $G$ holds $x \in \operatorname{Adjacent}(v)$ iff $\{v, x\} \in$ Edges $G$.

Let $X$ be a set. A simple graph is called a simple graph of $X$ if:

(Def. 9) Verticesit $=X$.

Let $X$ be a set. The functor CompleteSGraph $X$ yields a simple graph of $X$ and is defined by:

(Def. 10) CompleteSGraph $X=\{V ; V$ ranges over finite subsets of $X: \overline{\bar{V}} \leq 2\}$.

One can prove the following proposition 
(32) For every simple graph $G$ such that for all sets $x, y$ such that $x, y \in$ Vertices $G$ holds $\{x, y\} \in G$ holds $G=$ CompleteSGraph Vertices $G$.

Let $X$ be a finite set. One can check that CompleteSGraph $X$ is finite.

The following propositions are true:

(33) For every set $X$ and for every set $x$ such that $x \in X$ holds $\{x\} \in$ CompleteSGraph $X$.

(34) For every set $X$ and for all sets $x, y$ such that $x, y \in X$ holds $\{x, y\} \in$ CompleteSGraph $X$.

(35) CompleteSGraph $\emptyset=\{\emptyset\}$.

(36) For every set $x$ holds CompleteSGraph $\{x\}=\{\emptyset,\{x\}\}$.

(37) For all sets $x, y$ holds CompleteSGraph $\{x, y\}=\{\emptyset,\{x\},\{y\},\{x, y\}\}$.

(38) For all sets $X, Y$ such that $X \subseteq Y$ holds CompleteSGraph $X \subseteq$ CompleteSGraph $Y$.

(39) For every simple graph $G$ and for every set $x$ such that $x \in \operatorname{Vertices} G$ holds CompleteSGraph $\{x\} \subseteq G$.

Let $G$ be a simple graph. One can check that there exists a subset of $G$ which is simple graph-like. $G$.

Let $G$ be a simple graph. A subgraph of $G$ is a simple graph-like subset of

Let $G$ be a simple graph. The functor Complement $G$ yields a simple graph and is defined as follows:

(Def. 11) Complement $G=$ CompleteSGraph Vertices $G \backslash$ Edges $G$.

Let us observe that the functor Complement $G$ is involutive.

Next we state two propositions:

(40) For every simple graph $G$ holds Vertices $G=$ Vertices Complement $G$.

(41) Let $G$ be a simple graph and $x, y$ be sets. If $x \neq y$ and $x, y \in \operatorname{Vertices} G$, then $\{x, y\} \in$ Edges $G$ iff $\{x, y\} \notin$ Edges Complement $G$.

\section{INDUCED SUbGRAPHS}

Let $G$ be a simple graph and let $L$ be a set. The subgraph induced by $G$ yielding a subset of $G$ is defined by:

(Def. 12) The subgraph induced by $G=G \cap 2^{L}$.

Let $G$ be a simple graph and let $L$ be a set. Observe that the subgraph induced by $G$ is simple graph-like.

Next we state two propositions:

(42) For every simple graph $G$ holds $G=$ the subgraph induced by $G$.

(43) For every simple graph $G$ and for every set $L$ holds the subgraph induced by $G=$ the subgraph induced by $G$. 
Let $G$ be a finite simple graph and let $L$ be a set. Observe that the subgraph induced by $G$ is finite.

Let $G$ be a simple graph and let $L$ be a finite set. One can check that the subgraph induced by $G$ is finite.

One can prove the following three propositions:

(44) For all simple graphs $G, H$ such that $G \subseteq H$ holds $G \subseteq$ the subgraph induced by $H$.

(45) For every simple graph $G$ and for every set $L$ holds Vertices (the subgraph induced by $G$ ) $=$ Vertices $G \cap L$.

(46) For every simple graph $G$ and for every set $x$ such that $x \in \operatorname{Vertices} G$ holds the subgraph induced by $G=\{\emptyset,\{x\}\}$.

\section{Clique, Clique Number, Clique Cover}

Let $G$ be a simple graph. We say that $G$ is a clique if and only if:

(Def. 13) $G=$ CompleteSGraph Vertices $G$.

The following propositions are true:

(47) Let $G$ be a simple graph. Suppose that for all sets $x, y$ such that $x \neq y$ and $x, y \in$ Vertices $G$ holds $\{x, y\} \in \operatorname{Edges} G$. Then $G$ is a clique.

(48) $\{\emptyset\}$ is a clique.

Observe that there exists a simple graph which is a clique. Let $G$ be a simple graph. Note that there exists a subgraph of $G$ which is a clique.

Let $G$ be a simple graph. A clique of $G$ is a clique subgraph of $G$.

Next we state the proposition

(49) For every set $X$ holds CompleteSGraph $X$ is a clique.

Let $X$ be a set. One can check that CompleteSGraph $X$ is a clique.

Next we state two propositions:

(50) For every simple graph $G$ and for every set $x$ such that $x \in \operatorname{Vertices} G$ holds $\{\emptyset,\{x\}\}$ is a clique of $G$.

(51) Let $G$ be a simple graph and $x, y$ be sets. If $x, y \in \operatorname{Vertices} G$ and $\{x, y\} \in G$, then $\{\emptyset,\{x\},\{y\},\{x, y\}\}$ is a clique of $G$.

Let $G$ be a simple graph. Observe that there exists a clique of $G$ which is finite.

We now state two propositions:

(52) For every simple graph $G$ and for every set $x$ such that $x \in \bigcup G$ there exists a finite clique $C$ of $G$ such that Vertices $C=\{x\}$.

(53) For every a clique simple graph $C$ and for all sets $u, v$ such that $u$, $v \in$ Vertices $C$ holds $\{u, v\} \in C$. 
if:

Let $G$ be a simple graph. We say that $G$ has finite clique number if and only

(Def. 14) There exists a finite clique $C$ of $G$ such that for every finite clique $D$ of $G$ holds order $D \leq \operatorname{order} C$.

Let us note that there exists a simple graph which has finite clique number.

Let us observe that every simple graph which is finite also has finite clique number.

Let $G$ be a simple graph with finite clique number. The functor $\omega(G)$ yielding a natural number is defined as follows:

(Def. 15) There exists a finite clique $C$ of $G$ such that order $C=\omega(G)$ and for every finite clique $T$ of $G$ holds order $T \leq \omega(G)$.

We now state several propositions:

(54) For every simple graph $G$ with finite clique number such that $\omega(G)=0$ holds Vertices $G=\emptyset$.

(55) For every void simple graph $G$ holds $\omega(G)=0$.

(56) Let $G$ be a simple graph and $x, y$ be sets. If $\{x, y\} \in G$, then the subgraph induced by $G$ is a clique of $G$.

(57) For every simple graph $G$ with finite clique number such that Edges $G \neq$ $\emptyset$ holds $\omega(G) \geq 2$.

(58) For all simple graphs $G, H$ with finite clique number such that $G \subseteq H$ holds $\omega(G) \leq \omega(H)$.

(59) For every finite set $X$ holds $\omega$ (CompleteSGraph $X)=\overline{\bar{X}}$.

Let $G$ be a simple graph and let $P$ be a partition of Vertices $G$. We say that $P$ is clique-wise if and only if:

(Def. 16) For every set $x$ such that $x \in P$ holds the subgraph induced by $G$ is a clique of $G$.

Let $G$ be a simple graph. Observe that there exists a partition of Vertices $G$ which is clique-wise.

Let $G$ be a simple graph. A clique-partition of $G$ is a clique-wise partition of Vertices $G$.

Let $G$ be a void simple graph. Note that every partition of Vertices $G$ which is empty is also clique-wise. if:

Let $G$ be a simple graph. We say that $G$ has finite clique cover if and only

(Def. 17) There exists a clique-partition of $G$ which is finite.

One can verify that every simple graph which is finite also has finite clique cover.

Let $G$ be a simple graph with finite clique cover. Note that there exists a clique-partition of $G$ which is finite. 
Let $G$ be a simple graph with finite clique cover and let $S$ be a subset of Vertices $G$. One can verify that the subgraph induced by $G$ has finite clique cover.

Let $G$ be a simple graph with finite clique cover. The functor $\kappa(G)$ yielding a natural number is defined by:

(Def. 18) There exists a finite clique-partition $C$ of $G$ such that $\overline{\bar{C}}=\kappa(G)$ and for every finite clique-partition $C$ of $G$ holds $\kappa(G) \leq \overline{\bar{C}}$.

\section{Stable Set, Coloring}

Let $G$ be a simple graph and let $S$ be a subset of Vertices $G$. We say that $S$ is stable if and only if:

(Def. 19) For all sets $x, y$ such that $x \neq y$ and $x, y \in S$ holds $\{x, y\} \notin G$.

We now state two propositions:

(60) For every simple graph $G$ holds $\emptyset_{\text {Vertices } G}$ is stable.

(61) For every simple graph $G$ and for every subset $S$ of Vertices $G$ and for every set $v$ such that $S=\{v\}$ holds $S$ is stable.

Let $G$ be a simple graph. Observe that every subset of Vertices $G$ which is trivial is also stable.

Let $G$ be a simple graph. Note that there exists a subset of Vertices $G$ which is stable.

Let $G$ be a simple graph. A stable set of $G$ is a stable subset of Vertices $G$.

The following two propositions are true:

(62) For every simple graph $G$ and for all sets $x, y$ such that $x, y \in \operatorname{Vertices} G$ and $\{x, y\} \notin G$ holds $\{x, y\}$ is a stable set of $G$.

(63) For every simple graph $G$ with finite clique number such that $\omega(G)=1$ holds Vertices $G$ is a stable set of $G$.

Let $G$ be a simple graph. Note that there exists a stable set of $G$ which is finite.

One can prove the following proposition

(64) For every simple graph $G$ and for every stable set $A$ of $G$ holds every subset of $A$ is a stable set of $G$.

Let $G$ be a simple graph and let $P$ be a partition of Vertices $G$. We say that $P$ is stable-wise if and only if:

(Def. 20) For every set $x$ such that $x \in P$ holds $x$ is a stable set of $G$.

The following proposition is true

(65) For every simple graph $G$ holds SmallestPartition(Vertices $G$ ) is stablewise. 
Let $G$ be a simple graph. Note that there exists a partition of Vertices $G$ which is stable-wise. A coloring of $G$ is a stable-wise partition of Vertices $G$. We say that $G$ is finitely colorable if and only if:

(Def. 21) There exists a coloring of $G$ which is finite.

One can verify that there exists a simple graph which is finitely colorable.

Let us note that every simple graph which is finite is also finitely colorable.

Let $G$ be a finitely colorable simple graph. Note that there exists a coloring of $G$ which is finite.

We now state two propositions:

(66) Let $G$ be a simple graph, $S$ be a clique of $G$, and $L$ be a set. If $L \subseteq$ Vertices $S$, then the subgraph induced by $G$ is a clique of $G$.

(67) Let $G$ be a simple graph, $C$ be a coloring of $G$, and $S$ be a subset of Vertices $G$. Then $C\lceil S$ is a coloring of the subgraph induced by $G$.

Let $G$ be a finitely colorable simple graph and let $S$ be a set. One can check that the subgraph induced by $G$ is finitely colorable. The functor $\chi(G)$ yielding a natural number is defined as follows:

(Def. 22) There exists a finite coloring $C$ of $G$ such that $\overline{\bar{C}}=\chi(G)$ and for every finite coloring $C$ of $G$ holds $\chi(G) \leq \overline{\bar{C}}$.

One can prove the following three propositions:

(68) For all finitely colorable simple graphs $G, H$ such that $G \subseteq H$ holds $\chi(G) \leq \chi(H)$.

(69) For every finite set $X$ holds $\chi($ CompleteSGraph $X)=\overline{\bar{X}}$.

(70) Let $G$ be a finitely colorable simple graph, $C$ be a finite coloring of $G$, and $c$ be a set. Suppose $c \in C$ and $\overline{\bar{C}}=\chi(G)$. Then there exists an element $v$ of Vertices $G$ such that $v \in c$ and for every element $d$ of $C$ such that $d \neq c$ there exists an element $w$ of Vertices $G$ such that $w \in \operatorname{Adjacent}(v)$ and $w \in d$.

Let $G$ be a simple graph. We say that $G$ has finite stability number if and only if:

(Def. 23) There exists a finite stable set $A$ of $G$ such that for every finite stable set $B$ of $G$ holds $\overline{\bar{B}} \leq \overline{\bar{A}}$.

One can check that every simple graph which is finite also has finite stability number.

Let $G$ be a simple graph with finite stability number. Observe that every stable set of $G$ is finite.

Let us note that there exists a simple graph which is non void and has finite stability number.

Let $G$ be a simple graph with finite stability number. The functor $\alpha(G)$ yielding a natural number is defined as follows: 
(Def. 24) There exists a finite stable set $A$ of $G$ such that $\overline{\bar{A}}=\alpha(G)$ and for every finite stable set $T$ of $G$ holds $\overline{\bar{T}} \leq \alpha(G)$.

Let $G$ be a non void simple graph with finite stability number. One can check that $\alpha(G)$ is positive.

Next we state the proposition

(71) For every simple graph $G$ with finite stability number such that $\alpha(G)=1$ holds $G$ is a clique.

Let us observe that every simple graph which has finite clique number and finite stability number is also finite.

We now state four propositions:

(72) For every simple graph $G$ and for every clique $C$ of $G$ holds Vertices $C$ is a stable set of Complement $G$.

(73) For every simple graph $G$ and for every clique $C$ of Complement $G$ holds Vertices $C$ is a stable set of $G$.

(74) For every simple graph $G$ and for every stable set $C$ of $G$ holds the subgraph induced by Complement $G$ is a clique of Complement $G$.

(75) For every simple graph $G$ and for every stable set $C$ of Complement $G$ holds the subgraph induced by $G$ is a clique of $G$.

Let $G$ be a simple graph with finite clique number. One can check that Complement $G$ has finite stability number.

Let $G$ be a simple graph with finite stability number. Note that Complement $G$ has finite clique number.

We now state several propositions:

(76) For every simple graph $G$ with finite clique number holds $\omega(G)=$ $\alpha($ Complement $G)$.

(77) For every simple graph $G$ with finite stability number holds $\alpha(G)=$ $\omega($ Complement $G)$.

(78) For every simple graph $G$ holds every clique-partition of Complement $G$ is a coloring of $G$.

(79) For every simple graph $G$ holds every clique-partition of $G$ is a coloring of Complement $G$.

(80) For every simple graph $G$ holds every coloring of $G$ is a clique-partition of Complement $G$.

(81) For every simple graph $G$ holds every coloring of Complement $G$ is a clique-partition of $G$.

Let $G$ be a finitely colorable simple graph. One can check that Complement $G$ has finite clique cover.

Let $G$ be a simple graph with finite clique cover.

One can check that Complement $G$ is finitely colorable. 
One can prove the following propositions:

(82) For every finitely colorable simple graph $G$ holds $\chi(G)=$ $\kappa($ Complement $G)$.

(83) For every simple graph $G$ with finite clique cover holds $\kappa(G)=$ $\chi($ Complement $G)$.

\section{Mycielskian of a Graph}

Let $G$ be a simple graph. The functor Mycielskian $G$ yielding a simple graph is defined by the condition (Def. 25).

(Def. 25) Mycielskian $G=\{\emptyset\} \cup\{\{x\}: x$ ranges over elements of $\cup G \cup \cup G \times$ $\{\bigcup G\} \cup\{\cup G\}\} \cup$ Edges $G \cup\{\{x,\langle y, \cup G\rangle\} ; x$ ranges over elements of $\cup G, y$ ranges over elements of $\bigcup G:\{x, y\} \in \operatorname{Edges} G\} \cup\{\{\bigcup G,\langle x, \cup G\rangle\} ; x$ ranges over elements of $\bigcup G: x \in \operatorname{Vertices} G\}$.

We now state several propositions:

(84) For every simple graph $G$ holds $G \subseteq$ Mycielskian $G$.

(85) Let $G$ be a simple graph and $v$ be a set. Then $v \in$ Vertices Mycielskian $G$ if and only if one of the following conditions is satisfied:

(i) $v \in \bigcup G$, or

(ii) there exists a set $x$ such that $x \in \bigcup G$ and $v=\langle x, \bigcup G\rangle$, or

(iii) $v=\bigcup G$.

(86) For every simple graph $G$ holds Vertices Mycielskian $G=\bigcup G \cup \cup G \times$ $\{\bigcup G\} \cup\{\cup G\}$.

(87) For every simple graph $G$ holds $\bigcup G \in \bigcup$ Mycielskian $G$.

(88) For every void simple graph $G$ holds Mycielskian $G=\{\emptyset,\{\bigcup G\}\}$.

Let $G$ be a finite simple graph. Note that Mycielskian $G$ is finite.

The following propositions are true:

(89) For every finite simple graph $G$ holds order Mycielskian $G=2 \cdot \operatorname{order} G+$ 1.

(90) Let $G$ be a simple graph and $e$ be a set. Then $e \in \operatorname{Edges~Mycielskian~} G$ if and only if one of the following conditions is satisfied:

(i) $e \in \operatorname{Edges} G$, or

(ii) there exist elements $x, y$ of $\bigcup G$ such that $e=\{x,\langle y, \cup G\rangle\}$ and $\{x, y\} \in$ Edges $G$, or

(iii) there exists an element $y$ of $\bigcup G$ such that $e=\{\bigcup G,\langle y, \bigcup G\rangle\}$ and $y \in \bigcup G$.

(91) Let $G$ be a simple graph. Then Edges Mycielskian $G=$ Edges $G \cup\{\{x,\langle y$, $\bigcup G\rangle\} ; x$ ranges over elements of $\bigcup G, y$ ranges over elements of $\bigcup G$ : $\{x, y\} \in$ Edges $G\} \cup\{\{\bigcup G,\langle y, \cup G\rangle\} ; y$ ranges over elements of $\cup G$ : $y \in \bigcup G\}$. 
(92) For every finite simple graph $G$ holds size Mycielskian $G=3 \cdot \operatorname{size} G+$ order $G$.

(93) Let $G$ be a simple graph and $s, t$ be sets. Suppose $\{s, t\} \in$ Edges Mycielskian $G$. Then

(i) $\{s, t\} \in$ Edges $G$, or

(ii) $s \in \bigcup G$ or $s=\bigcup G$ but there exists a set $y$ such that $y \in \bigcup G$ and $t=\langle y, \cup G\rangle$, or

(iii) $t \in \bigcup G$ or $t=\bigcup G$ but there exists a set $y$ such that $y \in \bigcup G$ and $s=\langle y, \bigcup G\rangle$.

(94) For every simple graph $G$ and for every set $u$ such that $\{\bigcup G, u\} \in$ Edges Mycielskian $G$ there exists a set $x$ such that $x \in \bigcup G$ and $u=\langle x$, $\cup G\rangle$.

(95) For every simple graph $G$ and for every set $u$ such that $u \in \operatorname{Vertices} G$ holds $\{\langle u, \cup G\rangle\} \in$ Mycielskian $G$.

(96) For every simple graph $G$ and for every set $u$ such that $u \in \operatorname{Vertices} G$ holds $\{\langle u, \cup G\rangle, \bigcup G\} \in$ Mycielskian $G$.

(97) For every simple graph $G$ and for all sets $x, y$ holds $\{\langle x, \bigcup G\rangle,\langle y$, $\cup G\rangle\} \notin$ Edges Mycielskian $G$.

(98) For every simple graph $G$ and for all sets $x, y$ such that $x \neq y$ holds $\{\langle x$, $\cup G\rangle,\langle y, \cup G\rangle\} \notin$ Mycielskian $G$.

(99) For every simple graph $G$ and for all sets $x, y$ such that $\{\langle x, \bigcup G\rangle, y\} \in$ Edges Mycielskian $G$ holds $x \neq y$ but $x \in \bigcup G$ but $y \in \bigcup G$ or $y=\bigcup G$.

(100) For every simple graph $G$ and for all sets $x, y$ such that $\{\langle x, \bigcup G\rangle, y\} \in$ Mycielskian $G$ holds $x \neq y$.

(101) For every simple graph $G$ and for all sets $x, y$ such that $y \in \cup G$ and $\{\langle x, \cup G\rangle, y\} \in$ Mycielskian $G$ holds $\{x, y\} \in G$.

(102) For every simple graph $G$ and for all sets $x, y$ such that $\{x, y\} \in$ Edges $G$ holds $\{\langle x, \cup G\rangle, y\} \in$ Mycielskian $G$.

(103) For every simple graph $G$ and for all sets $x, y$ such that $x, y \in$ Vertices $G$ and $\{x, y\} \in$ Mycielskian $G$ holds $\{x, y\} \in G$.

(104) For every simple graph $G$ holds $G=$ the subgraph induced by Mycielskian $G$.

(105) Let $G$ be a simple graph and $C$ be a finite clique of Mycielskian $G$. If $3 \leq \operatorname{order} C$, then for every vertex $v$ of $C$ holds $v \neq \bigcup G$.

(106) For every simple graph $G$ with finite clique number such that $\omega(G)=0$ and for every finite clique $D$ of Mycielskian $G$ holds order $D \leq 1$.

(107) For every simple graph $G$ and for every set $x$ such that Vertices $G=\{x\}$ holds Mycielskian $G=\{\emptyset,\{x\},\{\langle x, \bigcup G\rangle\},\{\bigcup G\},\{\langle x, \bigcup G\rangle, \bigcup G\}\}$.

(108) For every simple graph $G$ with finite clique number such that $\omega(G)=1$ 
and for every finite clique $D$ of Mycielskian $G$ holds order $D \leq 2$.

(109) For every simple graph $G$ with finite clique number such that $2 \leq \omega(G)$ and for every finite clique $D$ of Mycielskian $G$ holds order $D \leq \omega(G)$.

Let $G$ be a simple graph with finite clique number. Note that Mycielskian $G$ has finite clique number.

We now state two propositions:

(110) For every simple graph $G$ with finite clique number such that $2 \leq \omega(G)$ holds $\omega($ Mycielskian $G)=\omega(G)$.

(111) For every finitely colorable simple graph $G$ there exists a coloring $E$ of Mycielskian $G$ such that $\overline{\bar{E}}=1+\chi(G)$.

Let $G$ be a finitely colorable simple graph. Observe that Mycielskian $G$ is finitely colorable.

We now state the proposition

(112) For every finitely colorable simple graph $G$ holds $\chi($ Mycielskian $G)=$ $1+\chi(G)$.

Let $G$ be a simple graph. The Mycielskian sequence of $G$ yields a many sorted set indexed by $\mathbb{N}$ and is defined by the condition (Def. 26).

(Def. 26) There exists a function $m_{1}$ such that

(i) the Mycielskian sequence of $G=m_{1}$,

(ii) $m_{1}(0)=G$, and

(iii) for every natural number $k$ and for every simple graph $G$ such that $G=m_{1}(k)$ holds $m_{1}(k+1)=\operatorname{Mycielskian} G$.

We now state two propositions:

(113) For every simple graph $G$ holds (the Mycielskian sequence of $G)(0)=G$.

(114) Let $G$ be a simple graph and $n$ be a natural number. Then (the Mycielskian sequence of $G)(n)$ is a simple graph.

Let $G$ be a simple graph and let $n$ be a natural number. Observe that (the Mycielskian sequence of $G)(n)$ is simple graph-like.

The following proposition is true

(115) Let $G, H$ be simple graphs and $n$ be a natural number. Then (the Mycielskian sequence of $G)(n+1)=$ Mycielskian (the Mycielskian sequence of $G)(n)$.

Let $G$ be a simple graph with finite clique number and let $n$ be a natural number. One can check that (the Mycielskian sequence of $G)(n)$ has finite clique number.

Let $G$ be a finitely colorable simple graph and let $n$ be a natural number. One can check that (the Mycielskian sequence of $G)(n)$ is finitely colorable.

Let $G$ be a finite simple graph and let $n$ be a natural number. Observe that (the Mycielskian sequence of $G$ ) $(n)$ is finite.

One can prove the following propositions: 
(116) Let $G$ be a finite simple graph and $n$ be a natural number. Then order (the Mycielskian sequence of $G)(n)=\left(2^{n} \cdot \operatorname{order} G+2^{n}\right)-1$.

(117) Let $G$ be a finite simple graph and $n$ be a natural number. Then size (the Mycielskian sequence of $G)(n)=3^{n} \cdot \operatorname{size} G+\left(3^{n}-2^{n}\right) \cdot \operatorname{order} G+((n+$ 1) block 3).

(118) Let $n$ be a natural number. Then $\omega($ the Mycielskian sequence of CompleteSGraph 2)(n)) $=2$ and $\chi(($ the Mycielskian sequence of CompleteSGraph 2$)(n))=n+2$.

(119) For every natural number $n$ there exists a finite simple graph $G$ such that $\omega(G)=2$ and $\chi(G)>n$.

(120) For every natural number $n$ there exists a finite simple graph $G$ such that $\alpha(G)=2$ and $\kappa(G)>n$.

\section{REFERENCES}

[1] Grzegorz Bancerek. Cardinal numbers. Formalized Mathematics, 1(2):377-382, 1990.

[2] Grzegorz Bancerek. The fundamental properties of natural numbers. Formalized Mathematics, 1(1):41-46, 1990.

[3] Grzegorz Bancerek. The ordinal numbers. Formalized Mathematics, 1(1):91-96, 1990.

[4] Grzegorz Bancerek. Tarski's classes and ranks. Formalized Mathematics, 1(3):563-567, 1990.

[5] Grzegorz Bancerek. Mizar analysis of algorithms: Preliminaries. Formalized Mathematics, 15(3):87-110, 2007, doi:10.2478/v10037-007-0011-x.

[6] Grzegorz Bancerek and Krzysztof Hryniewiecki. Segments of natural numbers and finite sequences. Formalized Mathematics, 1(1):107-114, 1990.

[7] Czesław Byliński. Functions and their basic properties. Formalized Mathematics, 1(1):55$65,1990$.

[8] Czesław Byliński. Some basic properties of sets. Formalized Mathematics, 1(1):47-53, 1990.

[9] Agata Darmochwał. Finite sets. Formalized Mathematics, 1(1):165-167, 1990.

[10] Frank Harary. Graph theory. Addison-Wesley, 1969.

[11] Rafał Kwiatek. Factorial and Newton coefficients. Formalized Mathematics, 1(5):887-890, 1990.

[12] J. Mycielski. Sur le coloriage des graphes. Colloquium Mathematicum, 3:161-162, 1955.

[13] Beata Padlewska. Families of sets. Formalized Mathematics, 1(1):147-152, 1990.

[14] Konrad Raczkowski and Paweł Sadowski. Equivalence relations and classes of abstraction. Formalized Mathematics, 1(3):441-444, 1990.

[15] Piotr Rudnicki and Lorna Stewart. The Mycielskian of a graph. Formalized Mathematics, 19(1):27-34, 2011, doi: 10.2478/v10037-011-0005-6.

[16] Andrzej Trybulec. Enumerated sets. Formalized Mathematics, 1(1):25-34, 1990.

[17] Andrzej Trybulec. Many sorted sets. Formalized Mathematics, 4(1):15-22, 1993.

[18] Andrzej Trybulec and Agata Darmochwał. Boolean domains. Formalized Mathematics, 1(1):187-190, 1990.

[19] Zinaida Trybulec. Properties of subsets. Formalized Mathematics, 1(1):67-71, 1990.

[20] Oswald Veblen. Analysis Situs, volume V. AMS Colloquium Publications, 1931. 